\title{
A REAL-TIME CONTROL APPROACH BASED ON INTELLIGENT VIDEO SURVEILLANCE FOR VIOLATIONS BY CONSTRUCTION WORKERS
}

\author{
Shengyu $\mathrm{GUO}^{1}$, Chaohua XIONG${ }^{2}$, Peisong GONG ${ }^{1}$ \\ ${ }^{1}$ School of Economics and Management and Institute of Management Science and Engineering, \\ China University of Geosciences, Wuhan, China \\ ${ }^{2}$ School of Civil Engineering and Mechanics and Institute of Construction Management, \\ Huazhong University of Science and Technology, Wuhan, China
}

Received 12 October 2017; accepted 09 January 2018

\begin{abstract}
The unsafe behavior of workers is the main object of construction safety management, in which violations require increased attention due to their pernicious consequences. However, existing studies have merely discussed violations separately from unsafe behaviors. To respond quickly to workers' violations on site, this study proposes a real-time control approach based on intelligent video surveillance. First, scenes reflecting unsafe behaviors are automatically acquired through camera-based behavior analysis technology. Meanwhile, the time corresponding to the construction phase is recorded. Second, the temporal association rule model of worker's unsafe behavior is constructed, and the rule "construction phase $\rightarrow$ unsafe behavior" is determined by the Apriori algorithm to identify target behaviors necessary for critical control in different construction phases. Finally, statistical process control is used to find the trends of violations with frequency and mass characteristics through the dynamic monitoring of target behavior. In addition, real-time alerts of these unsafe acts are produced simultaneously. A pilot study is conducted on the cross-river tunnel project in Wuhan city, Hubei, China, and the violations related to construction machineries is proven to be controllable. Thus, the proposed approach promotes behavioral safety management on construction since it effectively controls workers' violations by real-time monitoring and analysis.
\end{abstract}

Keywords: unsafe behavior, violation, association rule, statistical process control, intelligent video surveillance.

\section{Introduction}

The construction sector is one of the most dangerous job sectors worldwide (Zhu et al. 2016), and thus, construction safety management is arduous. After studying nearly 75,000 accidents, Heinrich et al. (1980) attributed $88 \%$ of safety accidents to the unsafe behavior of workers. Statistical results obtained by DuPont over the last 10 years also indicated that $96 \%$ of injuries were due to unsafe acts rather than unsafe conditions (McSween 2003). The unsafe behavior of workers is considered as a direct cause of accidents, making it one of the main research objects of construction safety management. For this research, unsafe behaviors refer to observable dangerous actions that may lead to accidents. The general unsafe behaviors of operators could be loosely classified as either errors or violations (Reason 1997). Errors represent the mental or physical activities of individuals that fail to achieve their intended outcome, whereas violations refer to the willful disregard for rules and regulations. Helmreich (2000) re- ported that over half the "errors" observed in line safety operations audit were due to violations and that those who violated procedures were 1.4 times more likely to commit other types of errors than those who did not. O'Dea and Flin (2001) also indicated that violation of existing rules and procedures was considered one of the most important factors that contribute to accidents. Therefore, finding ways to effectively control workers' violations is an important issue in behavioral safety.

Previous studies have mainly focused on the control of workers' unsafe behaviors, but few have separately focused on workers' violations. The cognitive causes of workers' unsafe behaviors are either conscious or unconscious. Violations represent the unsafe behaviors that workers know but are willfully executed anyway, which more frequently lead to serious construction accidents than errors. Jazayeri and Dadi (2017) introduced various construction safety management systems and discussed

*Corresponding author. E-mail: gsy.yu@foxmail.com 
their components. The behavioral element was regarded as a critical component usually controlled through traditional methods such as observation, interview, and training. Observation by employees is a time-consuming process on construction sites. Interview and training focus on changing the attitudes of workers that have subjective influences. Some safety management systems had mentioned a safety factor for improving safety behavior that hazards needed monitoring to identify trends. Behaviorbased Safety (BBS) emerged from the research of Skinner (1953); BBS is widely used as an effective method for behavior improvement, because the intervention process targets specific behavior for constructive change (Geller 2011). Although BBS interventions proved useful, its consistent effectiveness vanished with the removal of intervention (DeJoy 2005). Few contractors have voluntarily collected goals and feedback from individual workers over time and have conducted corresponding interventions due to the dynamic and temporary nature of the construction industry. Furthermore, safety models combining behavioral and cultural elements have been used in the construction industry because many unsafe behaviors can be attributed to poor construction safety culture. For instance, an operational excellence model was developed to evaluate and improve safety performance for construction organizations (Liu et al. 2015). Compared with the workers' unintentional errors on construction sites, violations can be effectively controlled (Cigularov et al. 2010). In addition, violations jeopardize organization-level safety outcomes, and thus, additional control is necessary (Zohar 2014). If symptoms of such behaviors toward change are discovered early, then the possibility of accident occurrence will greatly decrease. Controlling workers' violations is also necessary in achieving excellent safety performance, with operational excellence defined as doing the right thing, the right way, every time - even when no one is watching (Liu et al. 2017). Barabasi (2005) found that certain behaviors do not occur randomly over time and space, but have characteristics of burstiness. Similarly, violations display characteristics of frequency and mass in different construction phases, thus generating massive unsafe behavior data in a short time. Therefore, a data-driven approach could be considered to control workers' violations.

The data-driven approach combines data acquisition techniques and data mining algorithms. The use of intelligent video surveillance helped improve the productivity and safety on construction sites (e.g. Aguilar, Hewage 2013; Teizer et al. 2013) as it can automatically obtain scenes reflecting workers' unsafe behaviors (Guo et al. 2016). The development of data mining algorithms also supports the analysis and utilization of massive data. The use of the association rule is effective for massive data processing in finding items among attributes of a large database hidden in relationships (Sarawagi et al. 2000). Violations are usually related to the construction phase. The association rule can identify unsafe behaviors that frequently occur at each construction phase to determine target behaviors that require critical control. Statistical process control (SPC) determines whether changes in processes significantly influence outcomes, and thus, SPC is commonly used in quality management (Oakland 2007). By applying SPC to the monitoring process via intelligent video surveillance, the data of target behaviors is dynamically analyzed. Thus, the trends on workers' violations can be identified to produce real-time alerts before encountering serious consequences.

This study reviews existing research on the control of worker's unsafe behavior, intelligent video surveillance applications on construction safety management, and association rule and SPC usage in the construction industry. This study further introduces the proposed approach for real-time control of workers' violations, and its applications on a cross-river tunnel construction site in Wuhan city, Hubei, China.

\section{Literature review}

\subsection{Control of worker's unsafe behavior}

Some studies have focused on BBS to explore its sustained effect on behavior control. Zhang and Fang (2013) proposed a continuous BBS strategy that integrates BBS into management routines to continue improving the effect of behavioral intervention. Choudhry (2014) indicated that BBS can continue to function effectively only to fully mobilize the enthusiasm of subcontractors and workers in participating. Li et al. (2015) established an extension of BBS for construction called proactive behavior-based safety, combining traditional BBS management with novel information technology, including BIM and RFID, to enhance behavior control. Visualization techniques including 3D game engine and virtual reality (e.g. Sacks et al. 2013) were applied to the behavioral safety training of workers, and application results showed partial effects on unsafe behavior control. In view of the links between unsafe behaviors and safety culture, Liu et al. (2015) developed a safety model involving cultural and behavioral elements to assess and improve safety performance. As construction and project organizations became overregulated, violation management emerged as an issue (Love, Smith 2016). Cavazza and Serpe (2009) explored the role of safety climate in reducing the attitudinal ambivalence of workers toward using personal protective equipment (PPE). Their result indicated that good safety climate maintained a direct effect on violation. Lingard et al. (2016) identified the violations of workers through a participatory video process, the results of which can help establish how they may function safely. Alper and Karsh (2009) argued that minimal research about violations control in work settings exist. Whether BBS interventions or safety training is used, workers' unsafe behaviors are controlled from psychological aspects. Although implementations have improved owing to technical development, control effects have been greatly determined by workers' safety attitudes. Furthermore, available safety models combining behavioral and cultural factors explain the necessity of controlling worker's violation to some extent. 
However, few studies have focused on workers' violations in the construction industry, and existing ones have lacked control approaches.

\subsection{Application of intelligent video surveillance on construction safety management}

Several studies have examined how intelligent video surveillance could be used in construction. To address site safety issues, Shrestha et al. (2015) established a safety system to monitor whether workers properly use PPE, and Zhu et al. (2016) proposed novel Kalman filters to predict movements of workers and mobile equipment on construction sites. Han and Lee (2013) proposed a framework of vision-based unsafe action detection for behavior monitoring which can identify critical unsafe behavior. Guo et al. (2017) showed a trend in intelligent video surveillance on construction safety to obtain and analyze worker's unsafe behavior in real-time.

\subsection{Association rule and SPC usage in construction industry}

The association rule, which is used to discover interesting patterns in relational databases, has potential applications in many areas. Several studies have utilized this method to mine factors influencing the causation of accidents. These studies include that of Verma et al. (2014), who investigated and identified main contributing factors and root causes for the occurrence of incidents. Cheng et al. (2010) used the association rule in analyzing 1,347 accidents to identify potential hazards in Taiwan construction projects. Liao and Perng (2008) found through association rule mining that the effect of rain on the occurrence of fatalities is considerably significant. In terms of project quality management, Cheng et al. (2015) proposed a genetic algorithm-based approach to discover multi-level patterns of construction defects for quality improvement. The SPC method dynamically monitors significant changes in key indicators by using statistical tools; this method was introduced by Shewhart (1931). This method has been used in several aspects of construction with increasing data generated from work progress. Stewart and Spencer (2006) integrated SPC into a structured process-improvement strategy for quality monitoring of engineering materials to reduce waste. Aliverdi et al. (2013) presented an approach to monitor earned value indices by applying statistical quality control charts. In terms of construction safety, Wetzel and Thabet (2016) described a data collection method and an analysis method using the six sigma define function to develop standard safety attributes for facility management. Liu (2016) used SPC in building a control model to dynamically monitor key factors related to falling accidents. Isaac and Edrei (2016) utilized realtime tracking data to control the exposure of construction workers to safety risks that accumulate and change over time. In conclusion, the association rule is an effective tool in mining potential correlations of influencing factors, mostly used in the analysis of accident factors. With the realization of automatic data collection in the construction industry, the SPC method has great application potential in data utilization.

\section{Proposed approach for the real-time control of workers' violations}

As shown in Figure 1, the process of the proposed approach includes three main parts accomplished by collecting, analyzing and, utilizing data on worker's unsafe behavior:

1. The collection process pre-defines common unsafe behaviors from safety standards and operating instructions, considering the functions of camerabased behavior analysis technology. These behaviors can be automatically recognized through the intelligent monitoring videos, allowing scenes that reflect the unsafe behavior of workers to be obtained. Time is also simultaneously recorded to identify construction phases through the Gantt chart, which is used to record actual construction progress and to reflect ongoing work.

2. The analysis process identifies the association rule "construction phase $\rightarrow$ unsafe behavior" to analyze collected data. According to the rule, collected data are preprocessed to prepare a data set for mining. Apriori is the mining algorithm, which contains three parameters, namely, support, confidence, and lift. Effective strong association rules satisfying parameter requirements are located from frequent item sets to ascertain the target behaviors that require critical control in different construction phases.

3. In the utilization process, the SPC method is integrated into intelligent video surveillance to dynamically monitor workers' target behaviors. The trends of workers' violations with frequency and mass characteristics automatically and timely found in the process of monitoring. In addition, real-time alerts for accident prevention are produced simultaneously. The implementation of this method includes the following steps: (1) the unsafe behavior rate is taken as the control index, then a center line (CL) and an upper control limit (UCL) are set to generate the control chart; (2) Process Capability Indices (PCI) reveal process zones based on specification limits (SLs). To calculate this index, monitored unsafe behaviors are classified at different levels using a risk assessment method. The SLs of unsafe behavior rates are identified according to risk level. If the PCI satisfies the requirement, then the phase changes from analysis to control in monitoring; (3) according to discrimination criteria detected in abnormal situations, the control chart is observed and analyzed to suppress the trends of violations with characteristics of frequency and mass. 


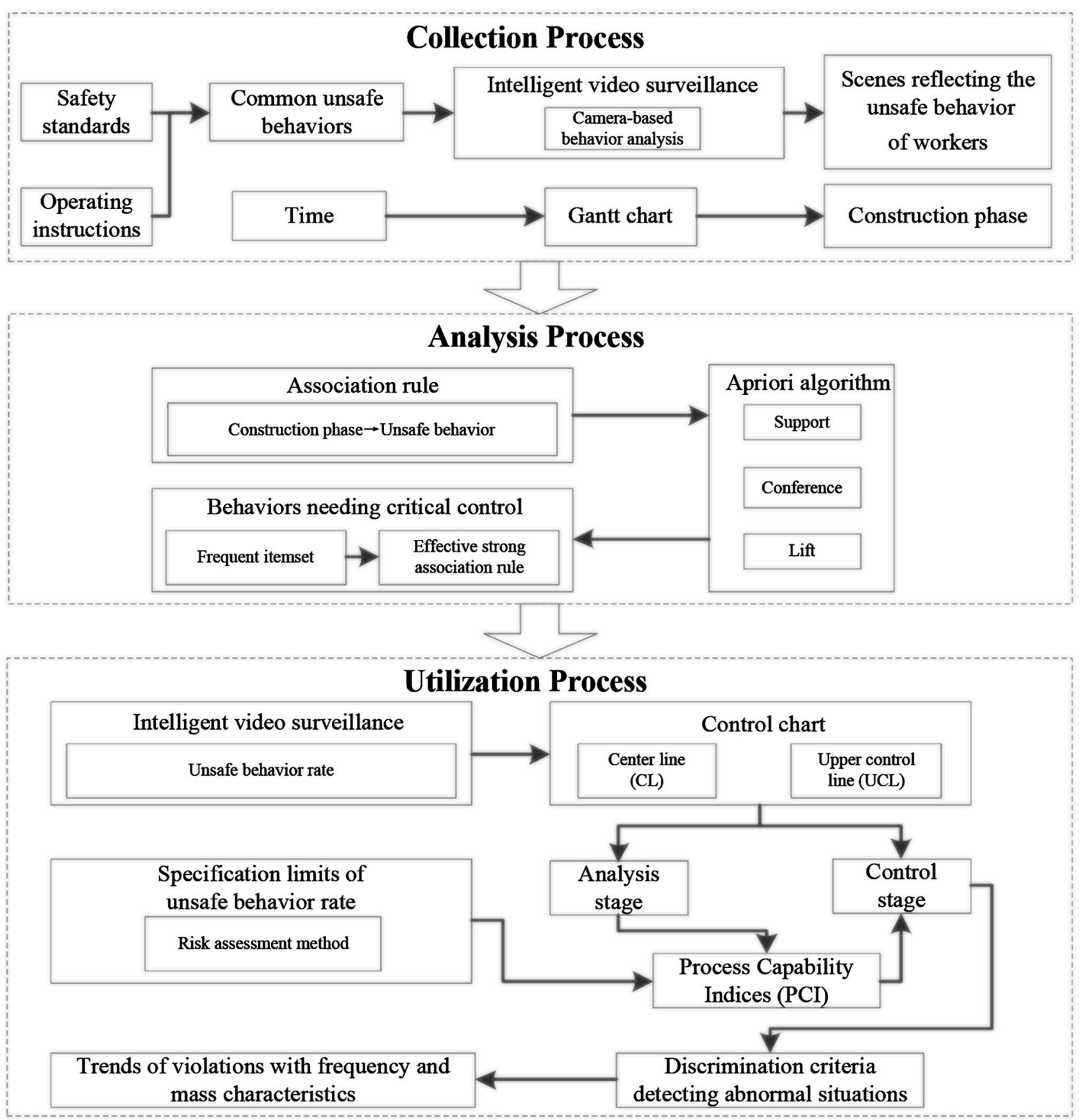

Figure 1. Process of the proposed approach

\subsection{Automatic data collection mechanism}

Two types of data are collected. A Big-Data-based platform was used to automatically obtain scenes reflecting the unsafe behaviors of workers from intelligent video surveillance (Guo et al. 2016). Construction phases could be identified through the Gantt chart according to the time when workers' unsafe behavior manifested.

\subsection{Time-related association rule mining of worker's unsafe behavior}

The association rule is expressed in $X \rightarrow Y$ form, where $X$ is the antecedent and $Y$ is the consequence. Data collected are preprocessed to build the mining database in "construction phase $\rightarrow$ unsafe behavior" form. The Boolean association rule is used, given that the data are discrete. Support, confidence, and lift are three important parameters which could be calculated by Eqns (1)-(3):

$$
\operatorname{Support}(X \cap Y)=\frac{\operatorname{num}(X \cap Y)}{\operatorname{num}(I)}=\frac{P(X \cap Y)}{P(I)},
$$

where: $P(X \cap Y)$ is the occurrence probability of item sets $X \cap Y$; $P(I)$ is the occurrence probability of database $I$; $P(I)=1 ; \operatorname{num}(X \cap Y)$ is the occurrence number of item sets $X \cap Y$; and num $(I)$ represents the occurrence number of Database $I$. 
Table 1. Behavioral risk assessment matrix

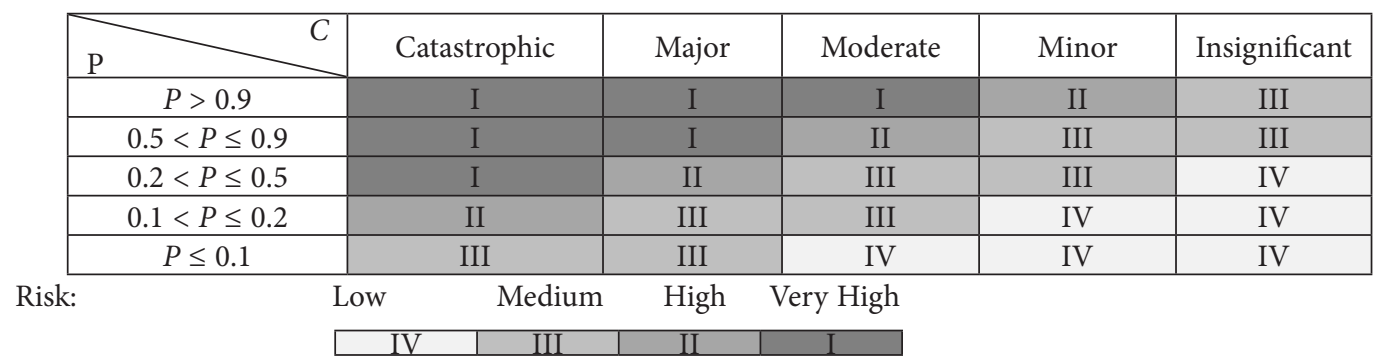

$$
\operatorname{Confidence}(X \rightarrow Y)=\frac{\operatorname{Support}(X \cap Y)}{\operatorname{Support}(X)}=\frac{P(X \cap Y)}{P(X)},
$$

where $P(X)$ is the occurrence probability of item $X$, and $\operatorname{Support}(X)$ is the support of item $X$.

$$
\operatorname{Lift}(X \rightarrow Y)=\frac{\text { Confidence }(X \rightarrow Y)}{\operatorname{Support}(Y)}=\frac{P(Y \mid X)}{P(Y)},
$$

where $P(Y)$ is the occurrence probability of item $Y$; $P(Y \mid X)$ is the occurrence conditional probability of item $Y$ under the condition that item $X$ exists; and $\operatorname{Support}(Y)$ is the support of item $Y$.

Apriori algorithm is employed to determine the rules (Agrawal et al. 1996). The process that uses the Apriori algorithm includes three steps: (1) Item sets with greater support than the minimum (threshold value set by the user) are determined to consist frequent item sets; (2) strong rules are determined from frequent item sets, wherein items with strong rules meet not only the demand for minimal support but also for minimum confidence; (3) effective strong association rules are verified by lift, with Lift $(X \rightarrow Y)>1$ signifying effectiveness. Effective strong association rules reflect target behaviors that require critical control in certain construction phases. Concentrating on these behaviors is typical as they easily happen on site.

\subsection{SPC for workers' violations}

Intelligent video surveillance dynamically monitors workers' target behaviors. SPC is then used to analyze monitored data and to identify statistically significant variations that should be addressed. Workers' violations with frequency and mass characteristics exist in these variations. The assumption is that the measured characteristic of the process has a normal distribution when the process is under control due to natural sources of variation.

Considering that times and durations of target behavior can be recorded by intelligent video surveillance, unsafe behavior rate is defined as the proportion of time when these behaviors occur in one hour. The Single ValueMoving Range (X-MR) chart is adopted in this study to determine whether this rate is in a controlled state. Otherwise, these abnormal data fluctuations may greatly be caused by frequent and mass violations. In the ideal state, unsafe behavior rate is expected to be distributed symmetrically around the mean $(\mu)$ and relative to the standard deviation ( $\sigma$ ); approximately $99 \%$ of measurements are expected to be within $\mu \pm 3 \sigma$. Therefore, $C L$ is determined to be set at the position of mean $(\mu)$. In addition to CL, UCL is set at the position of $\mu+3 \sigma$ for process control. In the monitoring process, the X-MR chart is divided into two stages, namely, the analysis and control stages. The prerequisite for determining the control stage process is that PCI $C_{p}$ meets specific requirements. The PCI $C_{p}$ is computed as:

$$
C_{P}=\frac{S L_{S}-\mu}{3 \sigma}
$$

where $S L_{S}$ is the specification limits (SLs). Generally, $C_{p} \geq 1.67$ indicates that the process capability is excellent, that $1.33<C_{P} \leq 1.67$ indicates that the process capability is sufficient and that $1 \leq C_{P}<1.33$ shows that the process capability is acceptable. In the above three cases, the process can be regarded as entering the control stage. For this monitoring process, SLs serve as the limit of unsafe behavior rate, which is identified from the safety management requirements in an actual construction project. To obtain this index, different types of unsafe behavior are divided into several levels. Considering that worker's unsafe behavior is a kind of safety risk, the combination of assessments regarding the probability of unsafe behavior occurrence $(P)$ and the consequence caused by unsafe behavior $(C)$ creates a modified risk matrix. This matrix defines the relative level of behavioral risk (Table 1). Risk index $(R)$ is computed as:

$$
R=P^{\star} C \text {. }
$$

Risk matrix approach was initially developed by the Electronic System Center of the US Air Force in April, 1995 to assess risk in the life cycle of purchase projects (Paul et al. 1998). The original risk matrix (ORM) has good applicability in risk assessment but still exhibits biases because levels of probability and consequence are identified through a subjective calculation process. To reduce those biases, probability is divided into five categories by calculating the previously mentioned support parameter, and consequence is assessed according to factors including the casualties, property loss and accident extent. The definitions of the five categories are presented in Table 2. Another significant weakness of ORM is the non-meticulous classification of risk index, which only has three qualitative levels. As a result, different risks may at times share the 
Table 2. Definitions of the five consequence categories

\begin{tabular}{|l|l|}
\hline Consequence Category & \multicolumn{1}{c|}{ Definition } \\
\hline Catastrophic & $\begin{array}{l}\text { A kind of unsafe behavior that, upon occurrence, would cause an extremely serious accident with } \\
\text { numerous casualties and property loss, as well as the ability to achieve minimum acceptable requirements. }\end{array}$ \\
\hline Major & $\begin{array}{l}\text { A kind of unsafe behavior that, upon occurrence, would cause a serious accident with many casualties } \\
\text { and property loss. Secondary requirements may be achieved. }\end{array}$ \\
\hline Moderate & $\begin{array}{l}\text { A kind of unsafe behavior that, upon occurrence, would cause a moderate accident with some } \\
\text { casualties and property loss, but important requirements would still be met. }\end{array}$ \\
\hline Minor & $\begin{array}{l}\text { A kind of unsafe behavior that, upon occurrence, would cause a small accident with few casualties and } \\
\text { property loss, and most requirements would still be achieved. }\end{array}$ \\
\hline Insignificant & $\begin{array}{l}\text { A kind of unsafe behavior that, upon occurrence, would cause an incident without casualties and prop- } \\
\text { erty loss, and without any effect on the construction project. }\end{array}$ \\
\hline
\end{tabular}

Table 3. Specific information about four discrimination criteria

\begin{tabular}{|l|c|l|}
\hline \multicolumn{1}{|c|}{ Discrimination criteria } & $\begin{array}{c}\text { Probability of } \\
\text { occurrence }\end{array}$ & \multicolumn{1}{c|}{ Rule } \\
\hline Points exceed UCL (i) & $0.27 \%$ & Real-time alerts are provided at points exceeding UCL \\
\hline Six successive points display consistent increase (ii) & $0.273 \%$ & $\begin{array}{l}\text { Real-time alerts are provided at the last of the six } \\
\text { points }\end{array}$ \\
\hline Two out of three consecutive points exceed $\mu+2 \sigma$ (iii) & $0.066 \%$ & $\begin{array}{l}\text { Real-time alerts are provided at the last of the three } \\
\text { points }\end{array}$ \\
\hline Four out of five consecutive points exceed $\mu+\sigma$ (iv) & $0.21 \%$ & $\begin{array}{l}\text { Real-time alerts are provided at the last of the five } \\
\text { points }\end{array}$ \\
\hline
\end{tabular}

same risk level in a specific case. Some studies have added extra risk index levels to reduce the vagueness of ORM to a certain extent (e.g. Ni et al. 2010; Zhou 2010; Isaac, Edrei 2016). After discussions with experts and on-site managers, four levels of behavioral risk are identified ranging from Low (IV) to Very High (I). Intervals of unsafe behavior rate corresponding to different levels are $1 \%-5 \%$ (I), $6 \%-10 \%$ (II), $11 \%-20 \%$ (III), and $21 \%-40 \%$ (IV). In practice, the specific value represented by SLs is determined by the level of safety management on construction sites.

In the control stage, a critical step is the detection of abnormal situations according to discrimination criteria, in which the trends of frequent and mass workers' violations exist. Out-of-control conditions are defined in Statistical Process Control Systems of GEIA Standard
(EIA557B 2006) addresses data points that (1) lie outside statistical control limits and (2) show significant changes, runs, trends, or patterns within statistical control limits. However, the discrimination criteria to be used in specific cases are determined by controllers. Montgomery (2009) discussed some sensitizing rules for the control chart and illustrated the definition of small probability as a critical factor in identifying discrimination criteria. Referring to previously defined discrimination criteria, re-definition of rules is required within the adaptation of the workers' violation control on site. Four items are finally determined from the eight discrimination criteria in the Standard of Shewhart Control Charts in China (GB/T 4091-2001). Other criteria are unsuitable because they cannot judge abnormally increasing trends of the unsafe behavior rate.

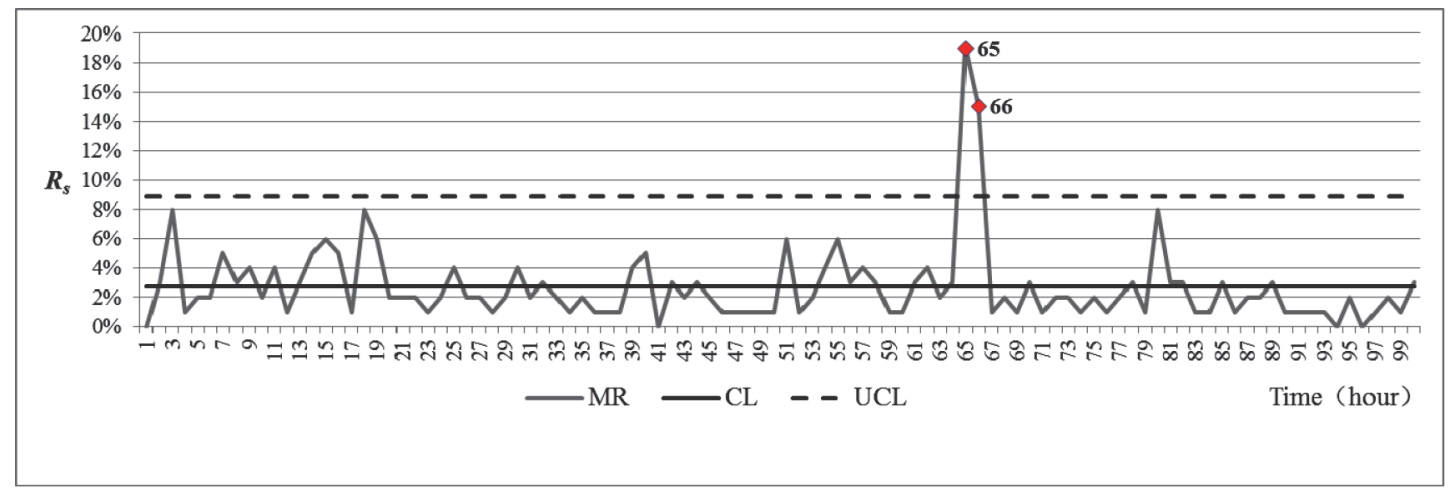

Figure 2. An example of the X-MR chart to control workers' violations 


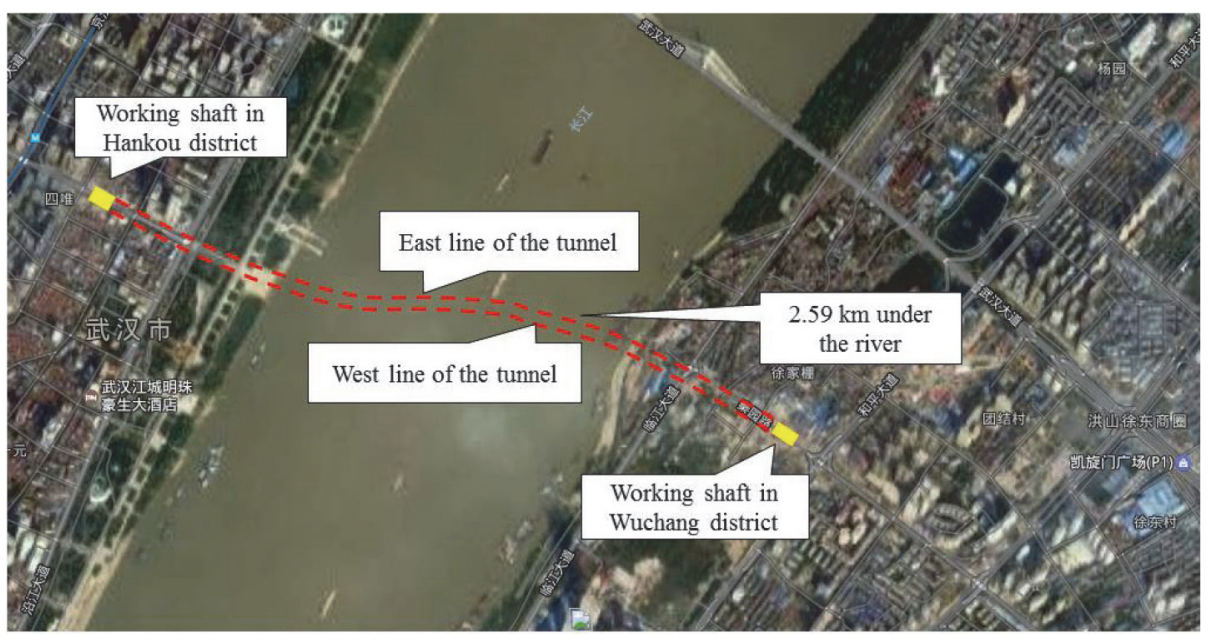

Figure 3. Satellite map of the cross-river tunnel project in Wuhan Metro Line 7

Small probability is defined as $0.27 \%$, indicating that these items can be considered as abnormal situations because the probability of their occurrence is extremely low. The rules for providing real-time alerts are provided corresponding to different items. Additional specific information are shown in Table 3.

Figure 2 presents a case identified in one of the tests conducted with the X-MR chart in which PCI meets the requirements. In Figure 2, the horizontal axis is the time axis wherein each number represents an hour, whereas the vertical axis represents the unsafe behavior rate monitored by intelligent video surveillance. The points with red marks in the X-MR chart indicate abnormal situations. The X chart shows that point 65 exceeds SLs, and points 13 and 18 exceed UCL. These points reflect that behavioral risk greatly increase, exceeding the acceptable level. In addition, points 12 and 13 are in violation of criterion (iii), point 14 violates criterion (iv), and points 49 and 50 violate criterion (ii). Although these points are under SLs and UCL, they reflect a dangerous proximity to UCL. All these abnormal situations reflect trends of violations, and thus, real-time alerts are provided to reduce behavioral risk. The MR chart is used to analyze non-random factors causing abnormal data fluctuations in the $\mathrm{X}$ chart. For instance, point 66 with the red mark violates criterion (i), which reflects that the alert produced for point 65 greatly reduces behavioral risk.

\section{Pilot study}

Wuhan, one of the major cities in Hubei, China, is executing a metro construction program with 12 lines under construction simultaneously. The cross-river tunnel project of Metro Line 7 is chosen as the application site. As shown in Figure 3, this tunnel is $4.6 \mathrm{~km}$ long, of which $2.59 \mathrm{~km}$ are constructed under a river. The construction task under a river is executed by two shield tunneling machines with diameters of $15.2 \mathrm{~m}$. Workers face a complicated construction environment in addition to extra potential behavioral risks caused by a highly congested construction site and complex geological and hydrological conditions. Workers with minimal safety training owing to tight construction schedule causes weak safety attitudes and frequent unsafe behaviors. Managers hope to reduce workers' observable unsafe behaviors, especially violations, through the real-time control approach. Therefore,

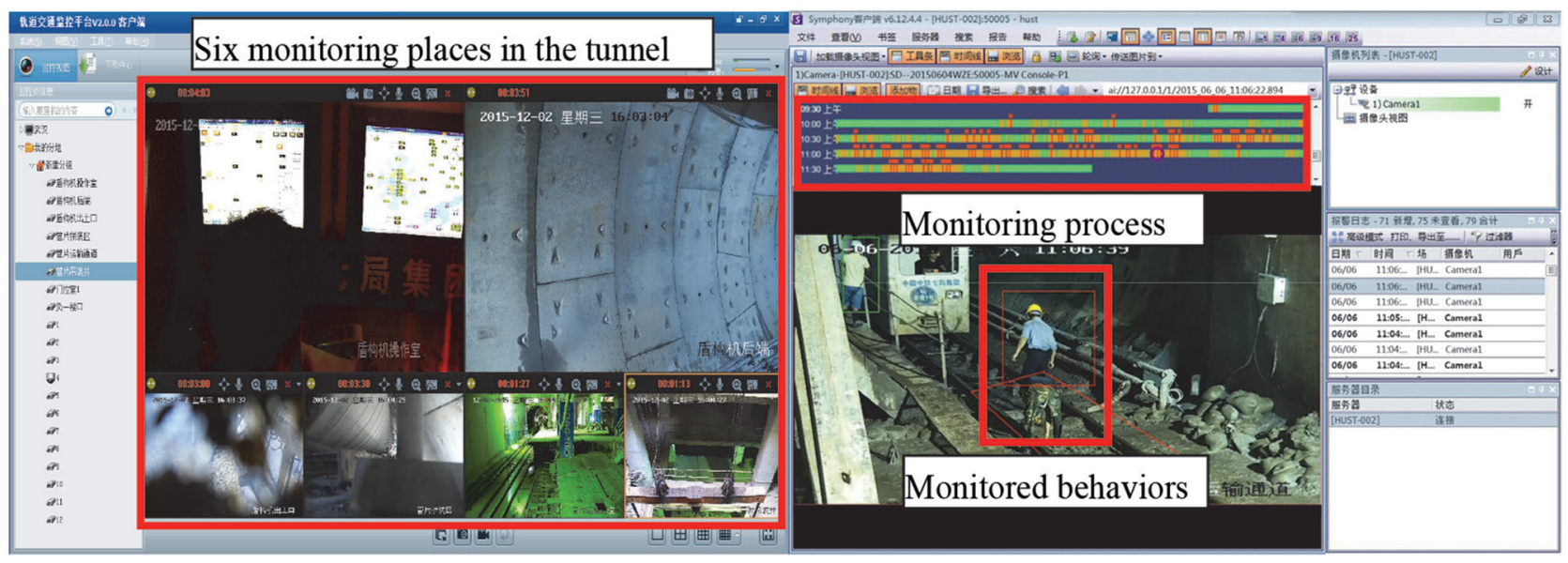

Figure 4. System interface of intelligent video surveillance 
a rapid reaction for behavioral risk is established to meet safety management goals.

\subsection{Data collection process}

Data collected through intelligent video surveillance in tunnel construction process includes three steps:

1) Camera positions were determined on site. The camera positions satisfied universal coverage of the construction site, as shown in Figure 4. Six cameras were separately located in soil removal places in the shield machine, lifting hole, tail of shield machine, duct piece assembly area, tunnel transportation zone, and shield machine operating room.

2) One camera-based behavior analysis function was chosen according to camera position. Camerabased behavior analysis technology includes functions such as intrusion detection, stay identification, and line-crossing detection. The function was pre-defined to automatically recognize possibly occurring unsafe behaviors within the scope of the monitor. Figure 4 demonstrates a possible unsafe

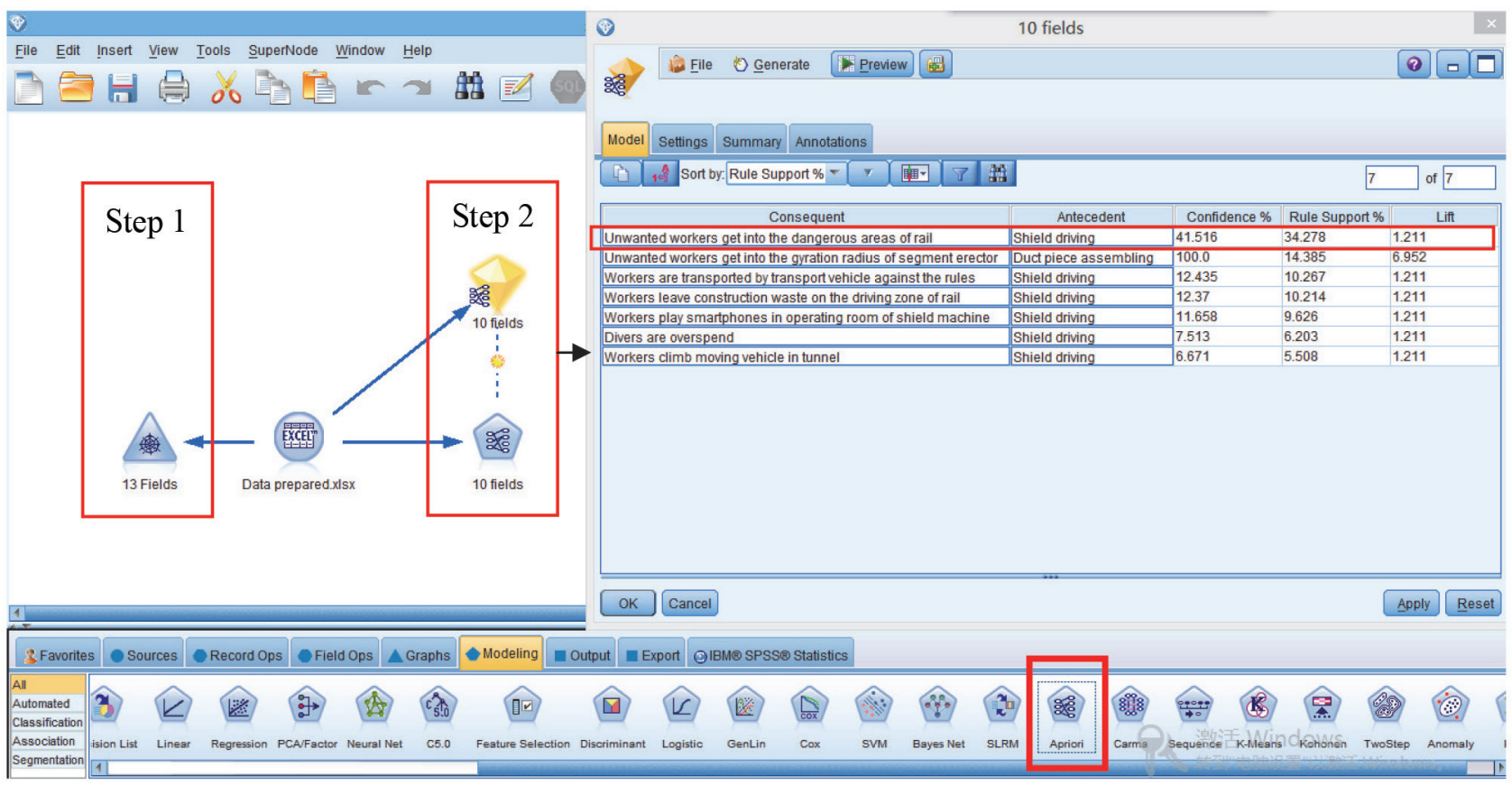

(a)

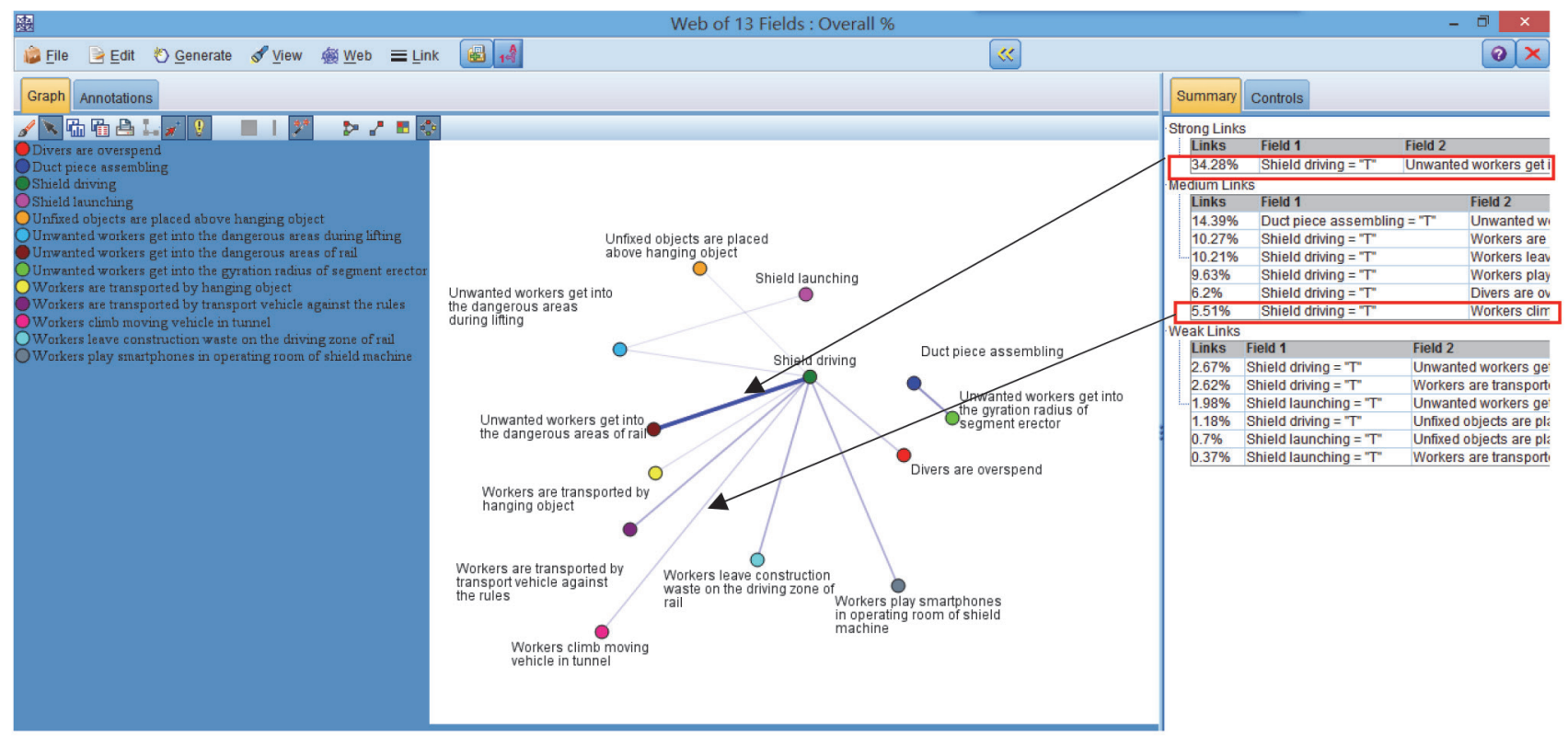

(b)

Figure 5. Visualization process of data mining by the Apriori algorithm in this study 

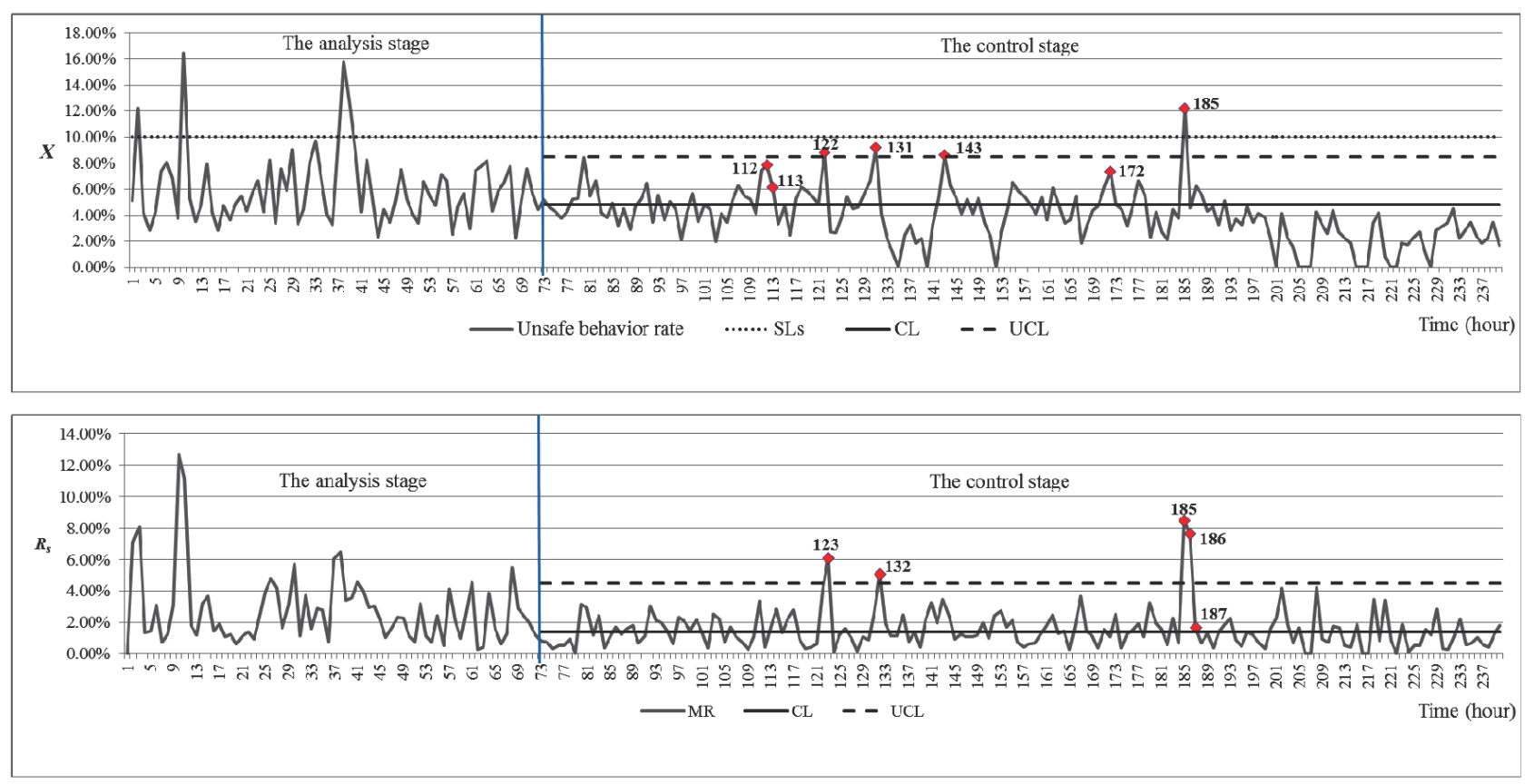

Figure 6. X-MR chart of monitored data collected over ten days

behavior in the tunnel transportation zone, where "unwanted workers get into the dangerous areas of rail". The behavior analysis function was selected as intrusion detection, which automatically recognized the entry of workers into these areas and exacted video scenes by observing the motions of workers.

3) The time when the unsafe behavior of workers happened was simultaneously recorded by camera. After finding the actual construction progress reflected by time in the Gantt chart, two types of data, namely, unsafe behavior and construction phase were obtained.

\subsection{Data analysis process}

Data collected in three months were preprocessed to form a data set for association rule mining. The preliminary analysis showed that workers mainly exhibited unsafe behavior during three construction phases, namely, shield launching, shield driving, and duct piece assembling. The machineries used in shield construction are either automatic or semi-automatic, thus injuries caused by machineries mostly occur in these phases. A total of 1,837 records of workers' unsafe behaviors related to machineries were analyzed through IBM SPSS Modeler 14.1 using Apriori algorithm. This process was illustrated in Figure 5 in two steps. Step 1 built a network reflecting links between unsafe behavior and the corresponding construction phase, from which the thickness of lines could intuitively indicate the degree of support shown in Figure 5 (b). For instance, the line between shield driving and unwanted workers get into the dangerous areas of rail is thicker than that between the former and workers climb a moving vehicle in tunnel because the first line is $34.28 \%$ bigger than the $5.51 \%$ support of the second line. Supports beyond 5\% represent frequent item sets. Thus, Step 2 further identified the strong rule from those item sets. Minimum support and confidence were both pre-defined as $30 \%$. As seen in Figure 5 (a), the strong rule was satisfied by "Shield driving $\rightarrow$ Unwanted workers get into the dangerous areas of rail". "Shield driving" was expressed as Item $X$, and "Unwanted workers get into the dangerous areas of rail" was expressed as Item $Y$, and then, $\operatorname{Lift}(X \rightarrow Y)=\frac{\text { Confidence }(X \rightarrow Y)}{\operatorname{Support}(Y)}=\frac{41.516 \%}{34.278 \%}=1.211>1$.

Therefore, this rule was proven effective. The result shows that this unsafe behavior required critical control for its frequent occurrence during this period.

\subsection{Data utilization process}

In the shield driving process, the target behavior identified above was dynamically monitored through intelligent video surveillance. The support calculated above was $34.28 \%$ and this behavior caused major consequence. Thus, this behavior was classified as Level II behavioral risk; SLs was preset at $10 \%$ to meet the requirement of safety management on site. Work was accomplished in three shifts a day for uninterrupted construction; data obtained in one day is a single batch. Figure 6 is the X-MR chart made using monitored data over 10 days, including the analysis and control stages which are as follows.

In the analysis stage, monitored data fluctuated largely on the first and second days, and the computed result was PCI $<1$ below the requirement. Thus, workers were warned to avoid this behavioral risk by on-site engineers. On the third day, the number was significantly reduced with PCI close to one. Monitored data began to fluctuate smoothly on the fourth day. The computed result was 
$\mathrm{PCI} \approx 1.43>1$, indicating that the monitoring process can proceed to the control stage the next day. The values $\mathrm{CL}=4.24$ and $\mathrm{UCL}=7.64$ were also calculated.

The control stage lasted from the fifth to the tenth day. The red points in the $\mathrm{X}$ chart of Figure 6 represent abnormal situations when real-time alerts were produced: points $122,131,143$, and 185 violated Criterion (i); point 172 violated Criterion (ii); and points 112 and 113 violated Criterion (iii). In hindsight, monitored data was abnormal in these points because of workers' violations. Even though workers had already known after undergoing safety training that such type of unsafe behavior may cause personal injuries, some still persisted for work for convenience or accessibility. Among the red points in the MR chart, abnormal data fluctuations of points 123, 132, and 186 show that unsafe behavior rate greatly decreases after real-time warning. As can be seen from the charts, unsafe behavior rate is maintained at a relatively low level, and data fluctuations remain smooth on the last two days. This result indicates that the real-time control approach can effectively reduce workers' violations.

\section{Discussions}

With the research trend of using data-driven methods to solve problems, a real-time control approach for workers' violations was proposed. Through monitoring via intelligent video surveillance, this approach opens the possibility of distinguishing the trends of frequent and mass workers' violations behind target behaviors in different construction phases. A pilot study showed positive feasibility results. The contributions of this research are twofold:

1. Time-related association rules of workers' unsafe behaviors are explored. On the basis of data obtained through intelligent video surveillance, the rules "construction phase $\rightarrow$ unsafe behavior" are mined by the Apriori algorithm. Critical behaviors can be identified for further monitoring in different construction phases.

2. An optimized method is used to identify workers' violations from the monitoring process. SPC is integrated into intelligent video surveillance to monitor target behaviors. Real-time alerts are produced to dynamically control violations when monitored data exceeds acceptable level, thereby reducing the occurrence of accidents on construction sites.

However, several limitations exist and should be acknowledged. Some factors, such as monitoring angle and foreground occlusion, influence intelligent video surveillance in accurately extracting scenes reflecting unsafe behavior of workers. On-site spatial influence is also not considered in identifying target behaviors in this study. Aside from construction phases, workers may behave differently in various locations. For instance, unsafe behavior easily occur near a foundation pit and near a crane are different. Future research should consider monitoring one kind of unsafe behavior from different angles to eliminate missing and false detection rates. Otherwise, the spatial-related association rules of workers' unsafe behaviors should be further analyzed to identify hazards in different areas of a construction site.

\section{Conclusions}

Violations are adverse to forming organizational safety climate in the construction industry, and the accidents they cause have a great negative effect on economy and society. Previous studies have merely isolated violations from unsafe behavior for the same control purpose from a psychological aspect. In actual cases, violations still occur even though workers know the dangers of such acts. To bridge the gap, this study proposed a real-time control approach to better control the on-site violations of workers. From theoretical points of view, behavioral safety management on construction is promoted. Behaviors that require critical control are mined through the association rule. These behaviors represent typical behavioral risks existing in different construction phases. Concentrating on these certain behaviors is necessary because violations involving them increase the probability of accident occurrence. With data monitored through intelligent video surveillance, change trends of these behaviors are analyzed through the SPC method. Real-time alerts are provided when abnormal data fluctuations appear. Symptoms of frequent and mass violations can be automatically and timely found through the use of this real-time control approach. Through this approach, reasons for violations can also be found in advance before they cause serious consequences. From a practical perspective, on-site video monitoring applications are improved. Video monitoring has become widely used with the development of lean construction. Manual checking tasks become increasingly heavy, costly, and tedious because data generated from videos considerably increased. Therefore, some videos are disused in practical applications. The proposed approach provides a solution to control workers' violations in the monitoring process. This solution can promote efficient and effective applications of on-site video surveillance. To achieve further contributions, defects in intelligent video surveillance should be resolved through repeated hardware and software tests to obtain valid data. Moreover, location-based technologies such as RFID should be considered to identify violations that frequently occur at specific places on site.

\section{Funding}

This work was supported by the Fundamental Research Funds for the Central Universities, China University of Geosciences (Wuhan) under Grant [number 170649]; the Research Foundation of Humanities and Social Sciences of Ministry of Education of China under Grant [number 15YJA630019]; the National Natural Science Foundation of China under Grant [number 71573237]; China Institute of Geo-environment Monitoring under Grant [number 0001212016CC60013]. 


\section{Disclosure statement}

Authors confirm that all of the content, figures (charts, photographs, etc.), and tables in the submitted manuscript work are original work created by the authors and no any competing financial, professional, or personal interests from other parties.

\section{References}

Agrawal, R.; Mannila, H.; Srikant, R.; Toivonen, H.; Verkamo, A. I. 1996. Fast discovery of association rules, Advances in Knowledge Discovery and Data Mining 12(1): 307-328.

Aguilar, G. E.; Hewage, K. N. 2013. IT based system for construction safety management and monitoring: C-RTICS2, Automation in Construction 35: 217-228.

https://doi.org/10.1016/j.autcon.2013.05.007

Aliverdi, R.; Moslemi Naeni, L.; Salehipour, A. 2013. Monitoring project duration and cost in a construction project by applying statistical quality control charts, International Journal of Project Management 31(3): 411-423.

https://doi.org/10.1016/j.ijproman.2012.08.005

Alper, S. J.; Karsh, B. T. 2009. A systematic review of safety violations in industry, Accident Analysis \& Prevention 41(4): 739-754. https://doi.org/10.1016/j.aap.2009.03.013

Barabasi, A.-L. 2005. The origin of bursts and heavy tails in human dynamics, Nature 435: 207-211. https://doi.org/10.1038/nature03459

Cavazza, N.; Serpe, A. 2009. Effects of safety climate on safety norm violations: exploring the mediating role of attitudinal ambivalence toward personal protective equipment, Journal of Safety Research 40(4): 277-283.

https://doi.org/10.1016/j.jsr.2009.06.002

Cheng, C.-W.; Lin, C.-C.; Leu, S.-S. 2010. Use of association rules to explore cause-effect relationships in occupational accidents in the Taiwan construction industry, Safety Science 48(4): 436-444. https://doi.org/10.1016/j.ssci.2009.12.005

Cheng, Y.; Yu, W. D.; Li, Q. 2015. GA-based multi-level association rule mining approach for defect analysis in the construction industry, Automation in Construction 51: 78-91. https://doi.org/10.1016/j.autcon.2014.12.016

Choudhry, R. M. 2014. Behavior-based safety on construction sites: A case study, Accident Analysis \& Prevention 70: 14-23. https://doi.org/10.1016/j.aap.2014.03.007

Cigularov, K. P.; Chen, P. Y.; Rosecrance, J. 2010. The effects of error management climate and safety communication on safety: A multi-level study, Accident Analysis \& Prevention 42(5): 1498-1506. https://doi.org/10.1016/j.aap.2010.01.003

DeJoy, D. M. 2005. Behavior change versus culture change: Divergent approaches to managing workplace safety, Safety Science 43(2): 105-129. https://doi.org/10.1016/j.ssci.2005.02.001

EIA557B Statistical Process Control Systems. Systems Management Council, 2006.

GB/T 4091-2001 Standard of Shewhart Control Charts. The General Administration of Quality Supervision, Inspection and Quarantine of the People's Republic of China, The Standardization Administration of the People's Republic of China, 2001.

Geller, E. S. 2011. Psychological science and safety large-scale success at preventing occupational injuries and fatalities, Current Directions in Psychological Science 20(2): 109-114. https://doi.org/10.1177/0963721411402667
Guo, H.; Yu, Y.; Skitmore, M. 2017. Visualization technologybased construction safety management: A review, Automation in Construction 73: 135-144.

https://doi.org/10.1016/j.autcon.2016.10.004

Guo, S. Y.; Ding, L. Y.; Luo, H. B.; Jiang, X. Y. 2016. A Big-Databased platform of workers' behavior: Observations from the field, Accident Analysis \& Prevention 93: 299-309.

https://doi.org/10.1016/j.aap.2015.09.024

Han, S.; Lee, S. 2013. A vision-based motion capture and recognition framework for behavior-based safety management, Automation in Construction 35: 131-141.

https://doi.org/10.1016/j.autcon.2013.05.001

Heinrich, H. W.; Petersen, D.; Roos, N. 1980. Industrial accident prevention. New York: McGraw-Hill.

Helmreich, R. L. 2000. On error management: lessons from aviation, BMJ: British Medical Journal 320: 781.

Isaac, S.; Edrei, T. 2016. A statistical model for dynamic safety risk control on construction sites, Automation in Construction 63: 66-78. https://doi.org/10.1016/j.autcon.2015.12.006

Jazayeri, E.; Dadi, G. B. 2017. Construction safety management systems and methods of safety performance measurement: A review, Journal of Safety Engineering 6(2): 15-28.

Li, H.; Lu, M.; Hsu, S. C.; Gray, M.; Huang, T. 2015. Proactive behavior-based safety management for construction safety improvement, Safety Science 75: 107-117. https://doi.org/10.1016/j.ssci.2015.01.013

Liao, C.-W.; Perng, Y.-H. 2008. Data mining for occupational injuries in the Taiwan construction industry, Safety Science 46(7): 1091-1102. https://doi.org/10.1016/j.ssci.2007.04.007

Lingard, H.; Pink, S.; Hayes, J.; McDermott, V. 2016. Using participatory video to understand subcontracted construction workers' safety rule violations, in Proceedings of the $32^{\text {nd }}$ Annual ARCOM Conference: Construction Work and the Worker. 5-7 September 2016, Manchester, UK, Association of Researchers in Construction Management, Vol. 1, 457-466.

Liu, H.; Jazayeri, E.; Dadi, G. B. 2017. Establishing the influence of owner practices on construction safety in an operational excellence model, Journal of Construction Engineering and Management 143(6): 04017005. http://dx.doi.org/10.1061/(ASCE)CO.1943-7862.0001292

Liu, H.; Jazayeri, E.; Dadi, G. B.; Maloney, W. F.; Cravey, K. J. 2015. Development of an operational excellence model to improve safety for construction organizations, in Proceedings of ICSC15: The Canadian Society for Civil Engineering $5^{\text {th }}$ International/1 $1^{\text {th }}$ Construction Specialty Conference, 2015, University of British Columbia, Vancouver, Canada. Vol. 96, 1-10. https://doi.org/10.14288/1.0076355

Liu, Z. 2016. Safety management analysis for construction industry: Statistical Process Control (SPC) approach: Thesis. The Faculty of the College of Business and Technology, Morehead State University.

Love, P. E.; Smith, J. 2016. Error management: implications for construction, Construction Innovation 16(4): 418-424. http://www.emeraldinsight.com/doi/abs/10.1108/CI-012016-0001

McSween, T. E. 2003. Values-based safety process: Improving your safety culture with behavior-based safety. New York: John Wiley \& Sons. https://doi.org/10.1002/0471721611

Montgomery, D. C. 2009. Introduction to statistical quality control. New York: John Wiley \& Sons.

Ni, H.; Chen, A.; Chen, N. 2010. Some extensions on risk matrix approach, Safety Science 48(10): 1269-1278. https://doi.org/10.1016/j.ssci.2010.04.005 
O’Dea, A.; Flin, R. 2001. Site managers and safety leadership in the offshore oil and gas industry, Safety Science 37(1): 39-57. https://doi.org/10.1016/S0925-7535(00)00049-7

Oakland, J. S. 2007. Statistical process control. Routledge.

Paul, R.; Garvey, P. R.; Lansdowne, Z. F. 1998. Risk matrix: an approach for identifying, assessing, and ranking program risks, Air Force Journal of Logistics 22(1): 16-23.

Reason, J. T. 1997. Managing the risks of organizational accidents. Ashgate Aldershot.

Sacks, R.; Perlman, A.; Barak, R. 2013. Construction safety training using immersive virtual reality, Construction Management and Economics 31(9): 1005-1017. http://dx.doi.org/10.1080/01446193.2013.828844

Sarawagi, S.; Thomas, S.; Agrawal, R. 2000. Integrating association rule mining with relational database systems: Alternatives and implications, Data Mining and Knowledge Discovery 4(2): 89-125. http://dx.doi.org/10.1023/a:1009887712954

Shewhart, W. A. 1931. Economic control of quality of manufactured products. New York: Macmillan.

Shrestha, K.; Shrestha, P. P.; Bajracharya, D.; Yfantis, E. A. 2015. Hard-hat detection for construction safety visualization, Journal of Construction Engineering, Article ID 721380. http://dx.doi.org/10.1155/2015/721380

Skinner, B. F. 1953. Science and human behavior. Simon and Schuster.

Stewart, R. A.; Spencer, C. A. 2006. Six-sigma as a strategy for process improvement on construction projects: a case study, Construction Management and Economics 24(4): 339-348. http://dx.doi.org/10.1080/01446190500521082

Teizer, J.; Cheng, T.; Fang, Y. 2013. Location tracking and data visualization technology to advance construction ironworkers' education and training in safety and productivity, Automation in Construction 35: 53-68.

https://doi.org/10.1016/j.autcon.2013.03.004

Verma, A.; Khan, S. D.; Maiti, J.; Krishna, O. B. 2014. Identifying patterns of safety related incidents in a steel plant using association rule mining of incident investigation reports, Safety Science 70: 89-98. https://doi.org/10.1016/j.ssci.2014.05.007

Wetzel, E. M.; Thabet, W. Y. 2016. Utilizing Six Sigma to develop standard attributes for a safety for facilities management (SFFM) framework, Safety Science 89: 355-368. https://doi.org/10.1016/j.ssci.2016.07.010

Zhang, M. Z.; Fang, D. P. 2013. A continuous behavior-based safety strategy for persistent safety improvement in construction industry, Automation in Construction 34: 101-107. https://doi.org/10.1016/j.autcon.2012.10.019

Zhou, J. 2010. SPA-fuzzy method based real-time risk assessment for major hazard installations storing flammable gas, Safety Science 48(6): 819-822.

https://doi.org/10.1016/j.ssci.2010.02.012

Zhu, Z.; Park, M.-W.; Koch, C.; Soltani, M.; Hammad, A.; Davari, K. 2016. Predicting movements of onsite workers and mobile equipment for enhancing construction site safety, Automation in Construction 68: 95-101. https://doi.org/10.1016/j.autcon.2016.04.009

Zohar, D. 2014. Safety climate: Conceptualization, measurement, and improvement. The Oxford handbook of organizational climate and culture. 